ThE AsTROPHYSICAL Journal, 369:L51-L53, 1991 March 10

(C) 1991. The American Astronomical Society. All rights reserved. Printed in U.S.A.

\title{
HUBBLE SPACE TELESCOPE WIDE-FIELD/PLANETARY CAMERA IMAGES OF SATURN
}

\author{
James A. Westrhal, ${ }^{1}$ William A. Baum, ${ }^{2}$ Tod R. Lauer, ${ }^{3}$ G. Edward Danielson, ${ }^{1}$ Douglas G. Currie, ${ }^{4}$ \\ S. P. Ewald, ${ }^{5}$ S. M. Faber, ${ }^{6}$ Edward J. Groth, ${ }^{7}$ J. JefF Hester, ${ }^{8}$ Robert M. Light, ${ }^{6}$ Roger LYNDS, $^{3}$ \\ Earl J. O’Neil, Jr., ${ }^{3}$ P. Kenneth Seidelmann, ${ }^{9}$ Edward J. Shaya, ${ }^{4}$ and Bradford A. Smith ${ }^{10}$ \\ Received 1990 October 18; accepted 1990 November 19
}

\begin{abstract}
Saturn was recorded in blue, green, and "red" light with the Wide-Field/Planetary Camera aboard the Hubble Space Telescope. Owing to relatively high signal levels over most of the planet's image, a dramatic improvement in the visibility of image detail was achieved by deconvolving the raw images, which had suffered severely from the spherical aberration of the HST optics. The deconvolved images are superior in quality to anything now achievable with ground-based telescopes. On Saturn, the polar hexagon seen by the Voyager spacecraft is still there, but some of the structure of the belts and zones has changed. The B-ring spokes were not visible.
\end{abstract}

Subject headings: image processing — planets: Saturn

\section{INTRODUCTION}

Saturn was among the targets selected to test the scientific sapability of the Hubble Space Telescope (HST) with its present optical handicap. Saturn differs from the other test targets by providing a high signal level over an area that is large in comparison with the point-spread function (PSF). Each point on the planet is smeared by spherical aberration as described by Burrows et al. (1991). The HST image of Saturn therefore consists of thousands of strongly overlapped PSFs, each with skirts about one-fifth as wide as the disk of the planet itself. Thus, the overall size of the HST PSF is similar to that of the PSF obtained with a ground-based telescope during average "seeing." The HST PSF differs, however, in having a sharp central spike containing about $15 \%$ of the signal, and the deconvolution process is able to take advantage of that.

The purpose of including Saturn among the test targets was to assess the possibility of monitoring time-variable phenomena on the planets with the HST in its present condition and with the aid of image deconvolution.

\section{OBSERVATIONS AND DATA REDUCTIONS}

HST images of Saturn were recorded on 1990 August 26 with the Wide-Field/Planetary Camera (WFPC). Passbands and exposure times are listed in Table 1, together with mean equatorial signal levels in the raw images (after bias subtraction). The WFPC was used in its wide field (f/12.9) mode to ensure that Saturn and its rings would all be contained within the area of one CCD (specifically, WF-2) with the

\footnotetext{
${ }^{1}$ Division of Geological and Planetary Sciences, California Institute of Technology, Pasadena, CA 91125.

${ }^{2}$ Astronomy Department, University of Washington, Seattle, WA 98105.

3 National Optical Astronomy Observatories, Tucson, AZ 85726.

${ }^{4}$ Department of Physics and Astronomy, University of Maryland, College Park, MD 20742.

5 Space Telescope Science Institute, Baltimore, MD 21218.

6 UCO/Lick Observatories, Board of Studies in Astronomy and Astrophysics, University of California, Santa Cruz, CA 95064.

${ }_{7}$ Physics Department, Princeton University, Princeton, NJ 08544.

8 Infrared Processing and Analysis Center, California Institute of Technology, Pasadena, CA 91125.

9 US Naval Observatory, Washington, DC 20390.

${ }^{10}$ Institute for Astronomy, University of Hawaii, Honolulu, H! 96822.
}

$H S T$ in a "point-and-shoot" mode. The Saturn observations were obtained with fixed telescope pointings while under gyro control, i.e., no guide stars were used. Owing to the shortness of the exposure times, there was no need for tracking Saturn's orbital motion. Moreover, HST planetary tracking capability was not yet operational.

The CCDs were preflashed so as to avoid deferred charge. Although the CCDs had not yet been ultraviolet-flooded to eliminate quantum efficiency hysteresis, that phenomenon was known from prelaunch tests to be unimportant within the area occupied by the Saturn image on WF-2.

At the time of observation, the geocentric distance of Saturn was $9.25 \mathrm{AU}$, the equatorial diameter of the disk was 17.9 , the outer diameter of the A ring was $40^{\prime \prime} .8$, the phase angle was 4.0 and was increasing, and the tilt of Saturn's pole was $24^{\circ} .0$. At the CCD, the image scale was $673 \mathrm{~km} \mathrm{pixel}^{-1}$, and the rings spanned just over 400 pixels. The relative motion of Saturn (including HST motion) was less than 0.02 pixels $\mathrm{s}^{-1}$ and therefore played no role. Neither did gyro drift, as confirmed by the absence of trailing in the image of a background star seen in these frames. Owing to 6 minute time gaps between exposures, however, disk rotations of approximately 3.5 (5.5 pixels at the equator) occurred between successive exposures. Thus, any cloud feature would not be found at the same location on blue, green, and red images.

Procedures described by Lauer (1989) were used for bias subtraction, flat-fielding, and cosmic-ray blemish removal. Onorbit flats from exposures of the bright Earth ("streak flats") were available for F439W; but for F547M and F718M, it was necessary to substitute streak flats of F555W and F785LP, respectively. Ground test data showed those to be satisfactory substitutions. Deconvolution was then performed on each of the three individual images using the algorithm of Lucy (1974). The PSF for F547M was taken from a recent HST-WFPC observation of NGC 188 (Holtzman et al. 1991); specifically, we mapped the spatial distribution of signal within the image of a relatively bright star that had been recorded in roughly the same location on the same CCD (WF-2) as the center of the Saturn image. For F439W and F718M, no PSF observations were yet available, so we had to fall back upon theoretical PSFs (Burrows et al. 1991). Each deconvolution was stopped after 40 iterations. 
TABLE 1

HST-WFPC OBSERVATIONS OF SATURN: 1990 August 26

\begin{tabular}{cccccc}
\hline \hline $\begin{array}{l}\text { UT } \\
(\mathrm{hr})\end{array}$ & $\begin{array}{c}\text { WFPC } \\
\text { Filter }\end{array}$ & $\begin{array}{c}\text { Effective } \\
(\mathrm{nm})\end{array}$ & $\begin{array}{c}\text { FWHM } \\
(\mathrm{nm})\end{array}$ & $\begin{array}{c}\text { Exposure Time } \\
(\mathrm{s})\end{array}$ & $\begin{array}{c}\text { Signal Level } \\
\text { (electrons pixel }\end{array}$ \\
\hline $12: 18: 52 \ldots \ldots \ldots$ & F439W & 435 & 51 & 0.4 & 8250 \\
$12: 24: 52 \ldots \ldots \ldots$ & F547M & 546 & 43 & 0.2 & 18000 \\
$12: 30: 52 \ldots \ldots \ldots$ & F718M & 719 & 60 & 0.2 & 15000 \\
\hline
\end{tabular}

\section{RESULTS AND CONCLUSIONS}

The deconvolved HST-WFPC images of Saturn, which are reproduced in Figure 1 (Plate L13), show more detail than the best ground-based images. Note Encke's Division in the A ring, for example. Although Encke's Division was discovered visually from the ground in 1837 , it has eluded efforts to record it photographically. In these HST images, the "division" stands out as a clear feature within the A ring. The visibility of features is further aided by combining the three deconvolved images of Figure 1 to produce the color picture in Figure 2 (Plate L14).

In contrast to the highly variable quality of ground-based imaging, HST imaging with deconvolution can be expected to remain uniform in quality. This means that, despite its present optical handicap, HST can successfully monitor time-variable phenomena on planets much better than can be done from the ground. Nor is it likely that projected improvements in ground-based imaging and image processing can consistently match $H S T$ performance in the foreseeable future.

$H S T$ has another advantage over ground-based telescopes: less scattered light. Part of that advantage is due to smoother and cleaner optical surfaces, and part is due to being outside Earth's atmosphere. This comparative freedom from scattered light, together with the large dynamic range of CCDs, makes it possible to record faint features alongside bright ones. In the digital image data, for example, the $\mathrm{C}$ ring (crepe ring) is well recorded in the same exposures that show very bright features in the A and B rings, but it is difficult to display both in a photographic reproduction.

These HST-WFPC observations yield two findings of scientific interest regarding Saturn: (1) The polar hexagon seen by Voyagers 1 and 2 in 1980-1981 (Godfrey 1988, 1990; Allison, Godfrey, \& Beebe 1990) still exists and was therefore not a transient phenomenon. In Figure 1 it can be seen best in the red (F718M) image, and it is also clearly visible in the color picture in Figure 2. (2) The latitude profile of belts and zones has changed somewhat from the profile seen by the Voyager spacecraft (Ingersoll et al. 1984). In Figure 3 (Plate L15) we compare our 1990 HST image of the northern hemisphere with a 1981 Voyager 2 image. The Voyager pixels have been binned to match our pixel size $(673 \mathrm{~km})$. To aid the comparison, we have rectified the Voyager image to the same polar tilt as ours, cut the images in half along the central meridian, and placed the halves in juxtaposition so that subtle differences in the belt and zone profile can be seen. We do not attempt here to interpret the differences in terms of atmospheric dynamics. Although the signal-to-noise $(\mathrm{S} / \mathrm{N})$ ratio of the Voyager image is enhanced by the binning of pixels, much of the $S / N$ difference in Figure 3 is due to the buildup of noise in the WFPC image during the deconvolution process, as discussed below. This, together with viewing geometry, may also account for the nondetection of the B-ring spokes in Figures 1 and 2.

The effectiveness of deconvolution is illustrated in Figure 4 by tracings across the east ansa of our F547M image, one before deconvolution was applied, and the other after 40 iterations of Lucy's (1974) algorithm. For both traces, five rows of pixels have been averaged.

The improvement in image quality through deconvolution was more dramatic for Saturn than for the other targets chosen to test present $H S T$ science capability, mainly because of the relatively high signal levels prevailing over most of the image, especially in the F547M and F718M passbands. The WFPC has a large dynamic range, $(\mathrm{S} / \mathrm{N})_{\max } \approx 200$. This Saturn result accords with the fact that deconvolution is a process in which one kind of image information is "traded" for another. During deconvolution, part of the original precision of pixel brightnesses in the raw image is "spent" in the process of redistributing image data spatially. A deconvolved image will therefore be inherently more "grainy" than the original image. The resulting degradation is acceptable if the original $S / N$ ratio was high enough, as it was in these Saturn images. Deconvolution will generally not be so effective in dealing with faint targets.

Future $H S T$ observations of planets made with the WFPC in its $\mathrm{f} / 30$ ("Planetary Camera") mode, instead of the $\mathrm{f} / 12.9$ mode used here, will permit 5 times more image integration time before reaching the same charge level per pixel. The $f / 30$ images will therefore contain more total signal (i.e., more image information), in addition to reducing that portion of the resolution loss which is due to image pixelization.

The spherical aberration of the HST can be "corrected" by the installation of a modified camera having an equal aberra-

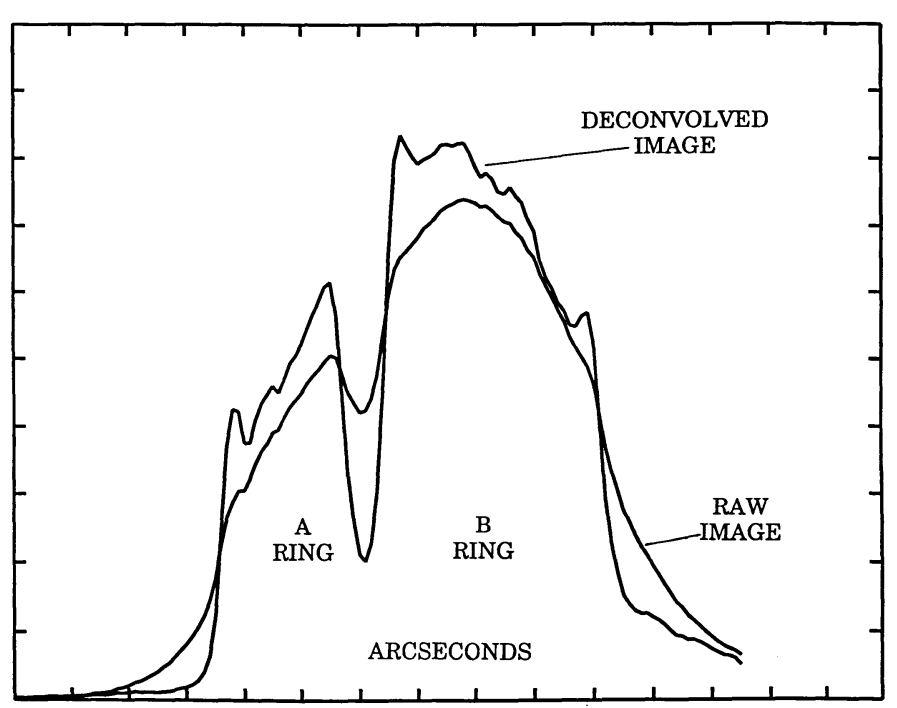

FIG 4-A plot of brightness across the east ansa of the A and $B$ rings on the F547M image. One curve represents the brightness distribution before deconvolution, while the curve with steeper slopes shows the distribution after deconvolution. Both curves are based on averaging five rows of pixels. 

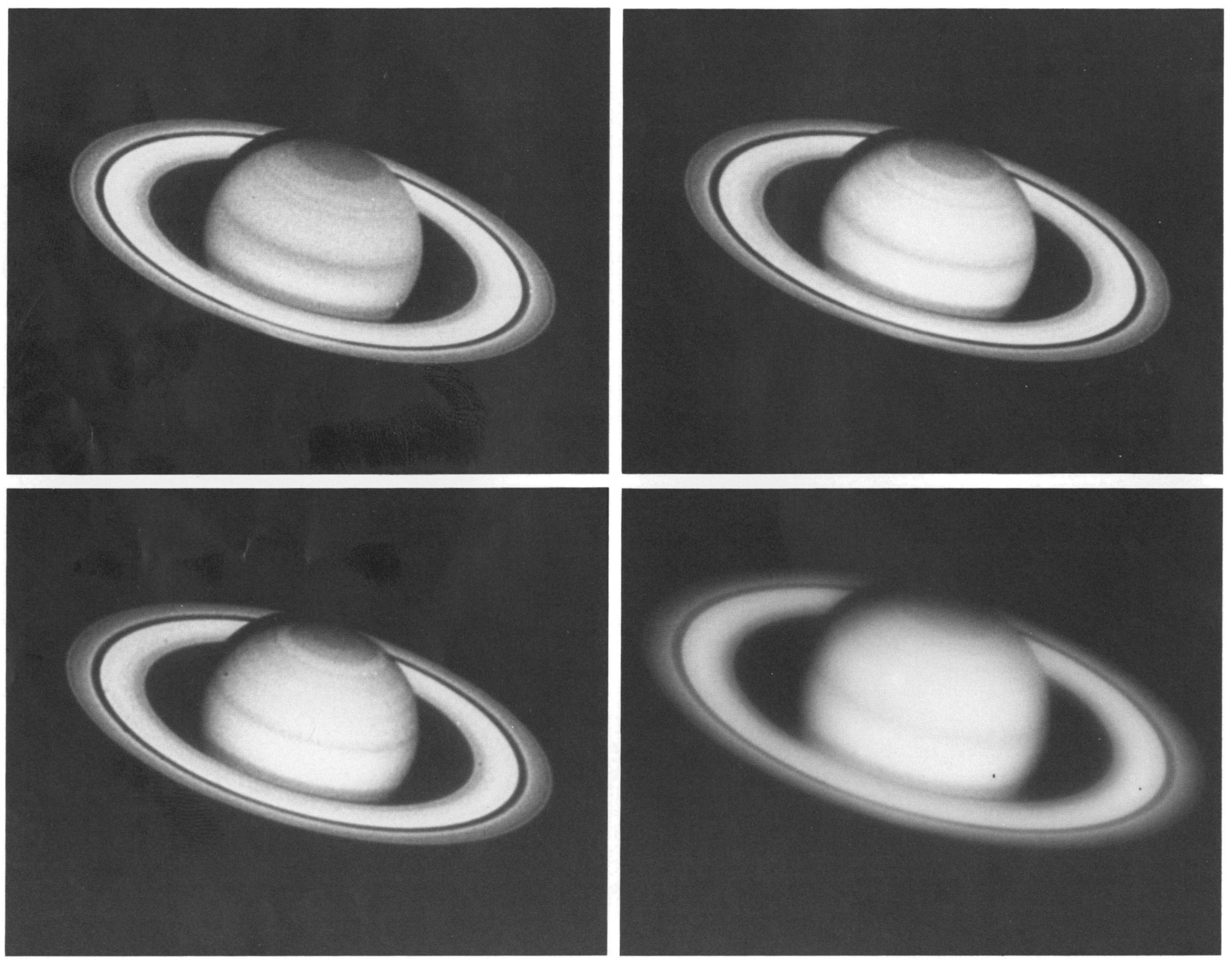

Fig. $1 c$

Fig. $1 d$ 


\section{PLATE L14}

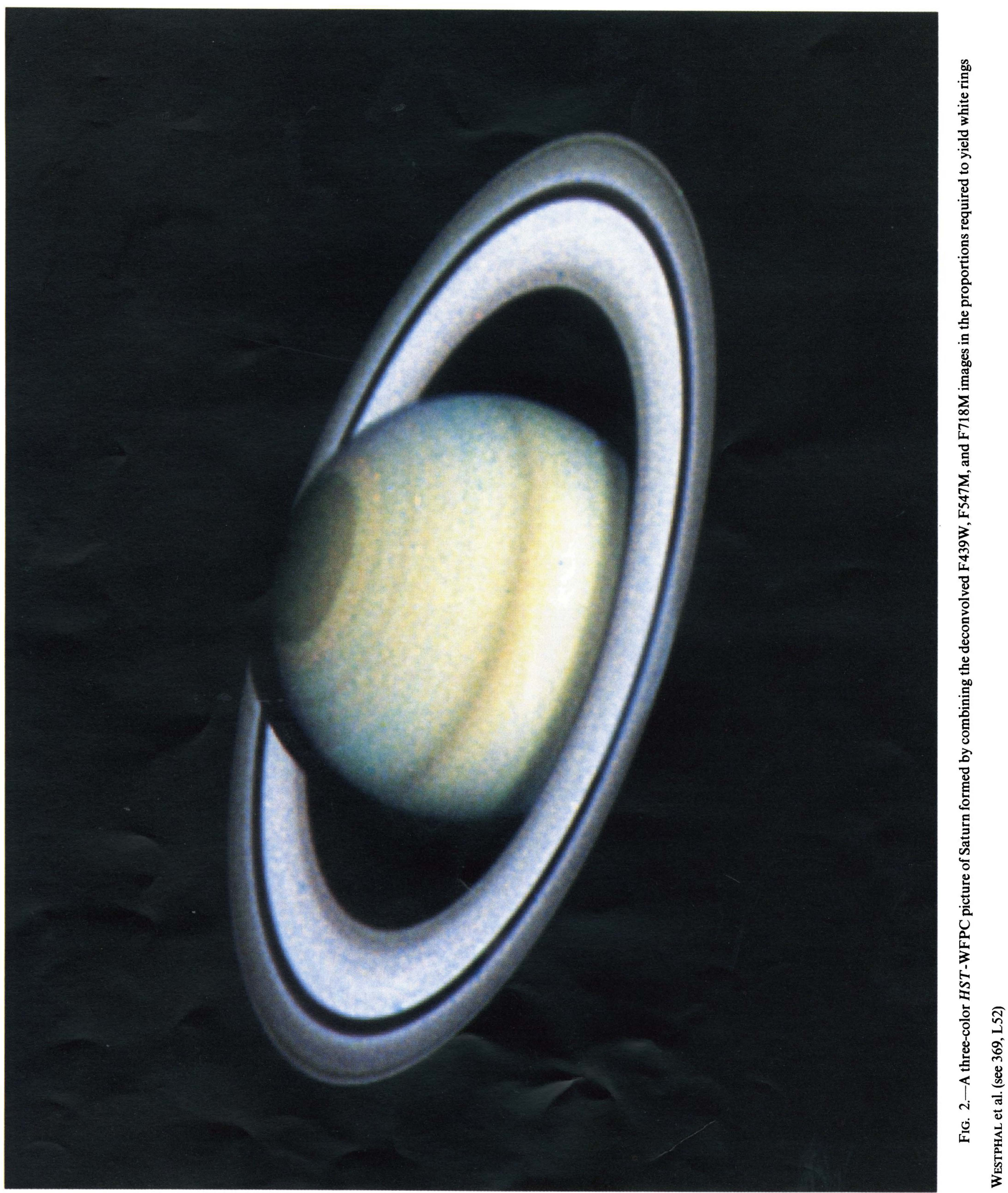

(c) American Astronomical Society - Provided by the NASA Astrophysics Data System 
PLATE L15

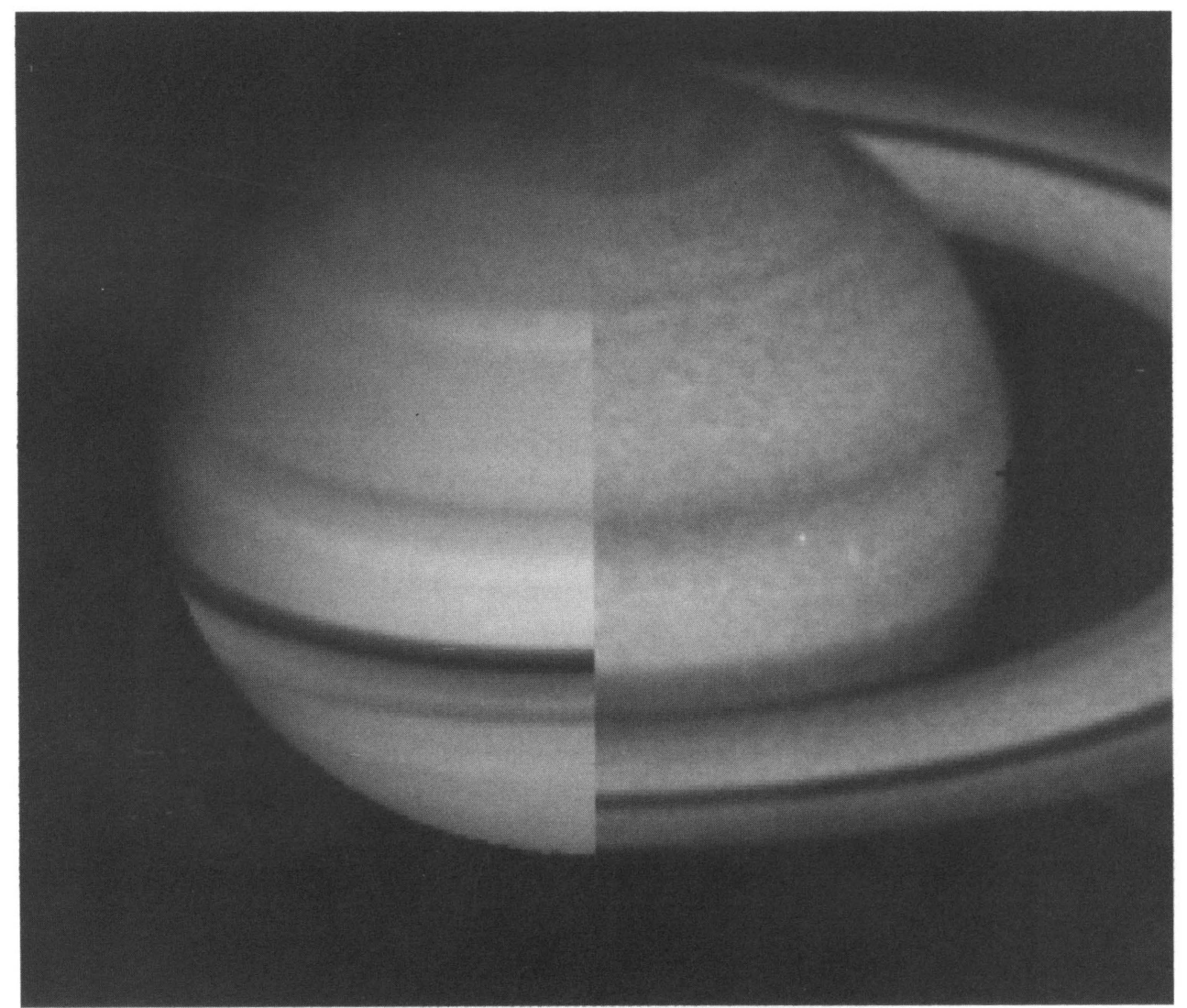

FIG. 3. - The 1990 structure of belts and zones in the northern hemisphere seen by HST (right) is compared with that observed in 1981 by Voyager 2 (left). The Voyager image has been rectified to the same polar tilt as our HST image. The dark equatorial band in the Voyager image is a shadow of the less tilted rings.

WeSTPHAL et al. (see 369, L52) 
tion of opposite sign. The main improvement for planetary imaging will be a higher $S / N$ ratio. This is because the $S / N$ degradation associated with deconvolution will no longer be present.

It is important to remember that the imaging of planets is a rather special case, not typical of most $H S T$-WFPC science. For most astronomical targets of high scientific interest, less signal is available to spend on deconvolution, so correction of the HST spherical aberration remains vital to most of the scientific goals for which WFPC was built.

\section{REFERENCES}

Allison, M., Godfrey, D. A., \& Beebe, R. F. 1990, Science, 247, 1061

Burrows, C. J., Holtzman, J. A., Faber, S. M., Bely, P. Y., Hasan, H., Lynds, C. R., \& Schroeder, D. 1991, ApJ, 369, L21

Godfrey, D. A. 1988, Icarus, 76, 335

1990 , Science, 247, 1206
Holtzman, J. A., et al. 1991, ApJ, 369, L35

Ingersoll, A. P., Beebe, R. F., Conrath, B. J., \& Hunt, G. E. 1984, in Saturn, ed. T. Gehrels \& M. S. Matthews (Tucson: University of Arizona Press), p. 195 Lauer, T. R. 1989, PASP, 101, 445

Lucy, L. B. 1974, AJ, 79, 745 